\title{
CDISC Schizophrenia Therapeutic Area User Guide Version 1.0
}

National Cancer Institute

\section{Source}

National Cancer Institute. CDISC Schizophrenia Therapeutic Area User Guide Version 1.0. NCl Thesaurus. Code C161452.

The 1.0 version of the CDISC Schizophrenia therapeutic area user guide. 